\title{
Difficulties of Learning the Italian Article from the Albanian Student
}

\author{
Msc. Entela Mustafaraj \\ Università “Ismail Qemali", Valona, Albania \\ entela.mustafaraj@gmail.com
}

\section{Doi:10.5901/jesr.2014.v4n4p258}

\begin{abstract}
The difficulties in the learning of the italian article is based on the fact that the category of the article results rather complex, as in the case of the italian article. A single morpheme has more than one function: gender, number and definition. Definite articles in albanian language practically don't exist, and because of this, in the first phases of acquisition, albanian students hardly learn the usage of the article. On the contrary, it exists the indefinite article 'një' after the unit of the indefinite form. The present article aims to determine relative problems of acquisition of the article from the albanian learners with the principal aim to confront albanian and italian system according to their functions. The method of investigation is followed by a contrasting analysis at a linguistic level. The interest directed at the presentation of the most important phenomenon indicated during the acquisition of the article from albanian learners, brings into light some key points in errors, except their possible cases.
\end{abstract}

Keywords: italian article, difficulty of learning, albanian students, confrontation between languages, analysis of errors.

\section{Introduzione}

L'acquisizione degli articoli italiani è generalmente una delle aree più problematiche per gli apprendenti albanesi nella prima fase di acquisizione dell'italiano come LS. In molte lingue, così come in italiano e in albanese, con l'uso dell'articolo definito 0 indefinito in un contesto, linguistico o extralinguistico, si segnala il significato della definitezza [-definitezza] del nome come categoria grammaticale. Dunque, l'articolo è il mezzo espressivo della categoria morfologica della definitezza e indefinitezza.

Le difficoltà dell'apprendimento risiedono nel fatto che gli articoli italiani sono parole assai complesse, perché variano tra forme preconsonantiche e forme prevocaliche della parola seguente. Nel caso di apprendenti albanesi, le difficoltà maggiori sono legate al fatto che gli articoli non sono trattati come una categoria linguistica a sé stante, pertanto sono chiamati morfemi o suffissi. Si noti che, durante il processo di apprendimento dell'italiano, gli studenti albanesi hanno la tendenza di omettere oppure di usare scorrettamente l'articolo all'interno del sintagma nominale. Quindi, durante il precorso dello studio dell'articolo italiano un albanofono deve compere alcune operazioni complesse per selezionare in modo corretto l'articolo: in primo luogo deve utilizzare l'articolo, saper scegliere tra l'articolo definito e quello indefinito, infine, accordare l'articolo in base al genere e al numero all'interno del SN.

\section{Gli Articoli in Italiano e in Albanese ${ }^{1}$}

Come già menzionato, il presente contributo si concentra sulle difficoltà dell'apprendimento degli articoli italiani dagli studenti albanesi di L1. Come in italiano anche in albanese esistono sia articoli definiti sia articoli indefiniti. È da notare però che il sistema degli articoli definiti è differente tra le due lingue. La distinzione riguarda la posizione tra articoli preposti, che appaiono prima del nome, e articoli postposti, che seguono il nome. Mentre l'articolo indefinito in italiano (uno) e in albanese (një) coincidono formalmente con il numerale 'uno' (Renzi 2001:386).

In italiano, l'articolo definito e indefinito, occupa la prima posizione nel sintagma nominale ed è spesso obbligatorio (Renzi 2001:374):

(1) il libro / la penna / *libro / *penna

(2) un ragazzo / una ragazza / *ragazzo / *ragazza

Riferendosi agli esempi riportati, sintatticamente, in italiano quasi tutti i nomi richiedono l'articolo, definito o indefinito, precedendoli spesso in modo diretto. In italiano, l'articolo definito ha varie forme sia per il genere che per il

\footnotetext{
${ }_{1}$ Nella grammatica tradizionale albanese, l'articolo definito va trattato come morfema, il quale svolge tra l'altro anche la funzione di definitezza. In assenza di un termine più adeguato, in questo lavoro farò riferimento convenzionalmente al termine 'articolo', a causa del trattamento della categoria dell'articolo italiano.
} 
numero, l'articolo indefinito invece varia nel genere, ma non nel numero. A volte si può usare l'articolo partitivo (dei, degli, delle) oppure il quantificatore (alcuni, certi) per esprimere il plurale di un concetto indeterminato. Si noti che l'articolo partitivo viene usato con i nomi di massa e in certi casi, quando il nome è al plurale, può non essere espresso:

(3) a. Renzo legge dei giornali.

b. Renzo legge alcuni giornali.

c. Renzo legge _ giornali dalla mattina alla sera.

Diversamente appare la situazione nella lingua albanese, dove il sistema degli articoli è più complicato rispetto all'italiano. Gli articoli non possono essere trattati come categoria morfologica. Inoltre, è importante sottolineare che, principalmente gli articoli in albanese sono trattati come una categoria a sé stante, ma con il passare del tempo gli articoli definiti, essendosi postposti al nome, hanno perso l'autonomia come parole distinte con determinate funzioni grammaticali e sono trasformate in morfemi formatori della categoria della determinatezza (Gramatika 1995:37).

Da questo punto di vista, gli articoli in base alla funzione occupano due posizioni diverse: proclitico ed enclitico. Topalli (2009:138) sottolinea che l'articolo enclitico è molto legato al nome precedente, prendendogli la categoria grammaticale del genere, numero e del caso. Quindi, svolgono un ruolo determinato o individualizzato. L'articolo proclitico invece ha altre funzioni, che generalmente non corrisponde con la funzione degli articoli. Dunque, essendo simile a un morfema, generalmente serve come formatore di nuove parole, però indica anche il genere, il numero e il caso dell'aggettivo articolato, il caso genitivo e concorda con il nome nel genere e nel numero.

Per quanto riguarda l'articolo indefinito një, la Gramatika e gjuhës shqipe (1995:38) afferma che in sostanza si tratta di un pronome indefinito utilizzato davanti ad un nome indeterminato. Secondo Newmark, Hubbard \& Prifti (1982:150-151) l'articolo indefinito një si usa generalmente quando il referente del nome è uno solo, non identificabile, ma soprattutto quando il nome ha un significato generale. Nella lingua albanese come in quella italiana non esistono articoli indefiniti per la forma del plurale. Però, l'albanese dispone altri mezzi sintattici per esprimere l'indefinitezza come ndonjë 'qualche' ca 'alcuni' njëfarë 'un certo' disa 'alcuni, alcune', i quali indicano che il referente del SN che introducono non sia presunto di essere identificabile all'ascoltatore.

(4) a. libri / miku / shtëpia

b. libri i nxënësit / dera e shtëpisë

'il libro / l'amico / la casa'

c. një djalë / një vajzë

'il libro dell'alunno / la porta della casa'

'un ragazzo / una ragazza'

Come si può osservare in (2a) l'articolo definito è un morfema enclitico al nome che morfologicamente serve a differenziare le sue categorie del genere, numero e caso. Mentre in (2b) l'articolo proclitico, rispettivamente i morfemi ' $i$ ' e ' $e$ ', il quale in alcuni casi non è legato al nome nel caso genitivo accordandosi nel genere e nel numero non con il nome davanti al quale si trova, ma con il nome che lo precede. In (2c) l'articolo indefinito një precede il nome al singolare, senza differenziare il genere e può marcare anche il caso (një djalë, një djali, prej një djali).

\section{Difficoltà di Apprendimento Dell'articolo Italiano}

L'articolo come elemento essenziale, che accompagna quasi tutti i nomi dell'italiano, è difficile da usare per un parlante non italiano. Come abbiamo osservato in precedenza, la scelta dell'articolo e il suo uso in modo corretto per un parlante non italiano comporta scelte complicate, soprattutto se gli apprendenti di L1 appartengono ad una lingua che tipologicamente è diversa dall'italiano. Nella scelta dell'articolo italiano un apprendente deve tenere presente tre categorie essenziali legati al SN italiano: la definitezza o [-definitezza], il genere e il numero.

Le difficoltà maggiori sono legate al fatto che la categoria dell'articolo determinativo italiano si esprime con forme diverse: il, la, lo, l', i, gli e le, rendendo il paradigma italiano più complesso e meno trasparente rispetto a quello albanese ed è probabile che ci vorrà più tempo e una maggiore esposizione all'input prima che si impari l'uso dei vari articoli italiani.

\subsection{L'espressione della definitezza e indefinitezza}

La definitezza o meno viene legata alla conoscenza che il parlante e l'ascoltatore hanno nell'identificare i referenti del SN al momento dell'enunciazione (Renzi 2001:379).

Lyons (1999:1) sostiene che un nome, in molte lingue, può contenere un elemento che abbia come ruolo quello di indicare la definitezza o meno del SN. Questo elemento può essere un articolo definito (il, lo, la) o indefinito (un, uno, una) come in italiano, oppure un suffisso definito $(-i,-u,-a) 0$ un articolo indefinito (një) come in albanese. Si noti che gli articoli, definito e indefinito, formano un'opposizione semantica tra l'un l'altro. Quindi, come osserva Serianni (1989:161162) la differenza tra i due tipi di articolo "non consiste propriamente nel fatto che il primo designa un nome in modo 
specifico e individuale e il secondo in modo generico" ma piuttosto nell'opposizione "noto/nuovo" oltre che nell'opposizione "classe/membro".

In seguito esamineremo in dettaglio l'uso dell'articolo definito e indefinito in italiano e in albanese:

(5) a. Ho preso in prestito il libro.

b. Ho preso in prestito un libro.

Come si vede, gli esempi in (5a)-(5b) riguardano la differenza sostanziale che vi è tra le due frasi: nel primo caso si tratta di un libro determinato, pertanto l'uso dell'articolo determinativo presuppone che esso è identificabile dal contesto oppure è stato già menzionato in precedenza tra parlante e ascoltatore; nel secondo caso invece il parlante non si riferisce a un libro in particolare, chi lo ascolta sa che si tratta di un libro qualsiasi.

(6) a. Shkuam me pushime në shtëpinë pranë liqenit.

Siamo andati in vacanza nella casa vicino al lago.

b. Shkuam me pushime në një shtëpi pranë liqenit.

Siamo andati in vacanza in una casa vicino al lago.

Si vede chiaro che, anche in albanese come in italiano, ci sono differenze fra l'articolo [+/-def.] così, in (6a) il sintagma në shtëpinë 'nella casa' viene usato con un significato specifico e individualizzato, mentre in (6b) il sintagma në një shtëpi 'in una casa' viene usato con un significato non-specifico e generico. La differenza fra le due frasi è chiara e tutto dipende dal elemento [+/-noto] all'ascoltatore e dal contesto in cui viene utilizzato.

Nella lingua italiana, l'espressione della [+/-def.] è veicolata dall'utilizzo di articoli definiti e indefiniti che secondo quanto conferma Renzi (2001:377-379) al livello semantico servono per differenziare tre tipi di sintagmi:

\section{$\mathrm{SN}$ determinato}

(7) Lorenzo porta fuori il cane. [+ noto / + specifico]

SN indeterminato specifico

(8) Domani arriva un nostro amico. [- noto / + specifico]

$\mathrm{SN}$ indeterminato non-specifico

(9) Vado a cena con un amico. [- noto / - specifico]

L'articolo definito si usa quando il parlante assume che l'ascoltatore sia in grado di individuare il referente al quale il SN rimanda. L'individuazione del referente può avvenire utilizzando strategie diverse: attraverso il rinvio al contesto linguistico 0 al contesto extralinguistico. In italiano l'articolo definito viene usato come segue:

a) uso anaforico: si ha quando un SN viene ripetuto dopo una precedente menzione: Roberto ha acquistato un appartamento. L'appartamento è nel palazzo che stanno costruendo vicino a casa mia.

b) uso cataforico: la determinatezza dipende dal contesto extralinguistico, cioè dalla supposizione del parlante che l'ascoltatore condivida le sue conoscenze: Ho perso il maglione che mi avevi regalato.

c) entità uniche: si ha con i SN che designano un referente unico: I/ sole era caldissimo; I/ cielo era pieno di nubi.

d) la classe: si ha con nomi che designano l'intera classe dei referenti ed ha quindi un valore generico: L'uomo è dotato di ragione; Il leone è il re della foresta.

e) nomi propri: i nomi propri danno origine a SN intrinsecamente determinati. La presenza o meno dell'articolo con i nomi propri non serve a opporre determinatezza a indeterminatezza: Milanol l'Italia.

f) con i possessivi: l'articolo definito viene usato anche in un SN che contiene un possessivo, tranne nel caso dei nomi di parentela al singolare: Ho preso la tua borsa; *ll nostro padre è severo.

È da osservare che l'italiano utilizza il dimostrativo ogniqualvolta che assolve la stessa funzione dell'articolo definito, quando secondo Renzi (1991:337) condividono la proprietà di definitezza con cui si informa l'ascoltatore che il referente cui si allude è [+noto,+specifico]:

(10) a. Quel professore d'italiano spiega bene.

b. Di chi è questa macchina?

L'articolo indefinito accompagna dei SN che non sono dati per identificati o per ben conosciuti (Renzi 2001:383). Kambarov (2008: 8-9) sottolinea che un nome con un articolo indefinito non si riferisce ad un particolare oggetto noto dal parlante e dall'ascoltatore. Va rilevato quindi, che l'articolo indefinito non assume che l'ascoltatore sia in grado di individuare il referente indicato dal SN, ma il parlante introduce nel discorso un refernte non identificato.

Prendendo spunto da alcune considerazioni fatte da Renzi (2001:382) sui SN indeterminati possiamo fare la distinzione semantica dei due tipi di [-definitezza] specifica e non-specifica, perché si rivelano elementi importanti durante l'acquisizione dell'articolo italiano:

(11)a. Ho comprato una bicicletta che cercavo da tempo.

b. Dall'altra parte della strada c'è un albergo.

L'articolo indefinito in entrambe le frasi non sono dati per identificati, però tra i due SN c'è una differenza: nel primo 
caso il SN è [+specifico, -noto] chi parla sa a quale bicicletta si riferisce, cioè si tratta di una bicicletta specifica ma non nota, nel secondo caso, invece, il SN è [-specifico, -noto] chi parla fa riferimento a un albergo qualsiasi.

\subsection{L'espressione di genere e numero}

Come è stato osservato sopra, l'articolo è parte integrale di un SN che oltre ad esprimere la definitezza e [-def], concorda con esso in genere e numero. Nella lingua italiano la desinenza del nome è un forte indicatore, ovvero indizio, sia di numero sia di genere. L'acquisizione del genere e del numero in italiano è assai difficile e problematico in quei nomi che in base a certi criteri di assegnazione risultano ambigui e arbitrari. Studiando le desinenze dei nomi italiani, si può osservare che la relazione tra numero e genere non è completamente trasparente. Si noti che i nomi della prima e seconda classe come in albero e bambina con la desinenza in -o e in -a al singolare e in - $i$ e in -e al plurale che sono univoche rispetto al genere, risultano meno problematici agli apprendenti in quanto sono le più frequenti e trasparenti.

Al contrario la terza classe come in ponte, limone, chiave, mare con la desinenza in -e al singolare e in -i al plurale pare più complessa, il che significa che partendo dalla vocale finale, è difficile prevedere il genere. Questi nomi appartengono sia al genere maschile sia al genere femminile. Ancora più difficile risulta la classe dei nomi che comprendono nomi di origine straniera come in leader $/ \mathrm{r}$, tram $/ \mathrm{m}$, sport $\mathrm{t}$, nomi con vocale finale accentata come in caucciü/ù, caffèlè, veritàlà; monosillabi re/e, gru/u, che rimangono invariabili sia al singolare che al plurale.

Per quanto riguarda i nomi maschili di origine greca che finiscono in -a al singolare e in -i al plurale come in problema, tema, diploma, fantasma, dei nomi femminili in -o al singolare e in - $i$ al plurale come in mano, dei nomi con la stessa desinenza al singolare e al plurale come in moto/o, gorilla/a, crisili e dei nomi con forma maschile al singolare e femminile al plurale come in uovola, paiola, miglio/a l'acquisizione si dimostra molto complicato, in cui gli apprendenti continuano a fare errori anche a livelli più avanzati.

\section{La Ricerca}

Nell'ambito dell'insegnamento dell'italiano LS in un corso di "Grammatica italiana" con studenti albanesi di L1 nella prima fase dell'acquisizione dell'italiano sono stati osservati alcuni errori relativi a uno scorretto uso degli articoli. L'obiettivo della ricerca è di evidenziare le fasi principali di acquisizione dell'articolo italiano e tentare di stabilire quale metodologia di insegnamento più adeguato sia per insegnare la categoria dell'articolo.

\subsection{Partecipanti}

II numero totale dei partecipanti è stato 20 studenti albanesi che frequentavano il corso della Grammatica italiana. Alcuni dei partecipanti coinvolti alla ricerca avevano frequentato, prima della loro iscrizione all'Università, un corso di lingua italiana, altri invece l'hanno studiata nelle scuole medie superiori e pochissimi durante la loro residenza in Italia. II gruppo dei partecipanti è stato in gran parte omogeneo per quanto riguarda l'età e il grado di istruzione.

\subsection{Materiali}

Lo studio principale consisteva di due attività: la prima attività è stata divisa in due produzioni scritte. È stato richiesto agli studenti di comporre due storie. Nella prima storia dovevano scegliere un tema libero, nella seconda, invece, il tema era obbligato e dovevano utilizzare un lessico semi guidato. L'argomento è stato scelto in modo tale da comprendere parole chiave: scuola, lezione, studente, professore, esame, diploma, esercizio, università appartenenti alle classi di parole descritte sopra. L'obiettivo era di verificare la conoscenza dell'acquisizione dell'articolo definito [-definito] in italiano. La seconda attività è stata concentrata su alcuni esercizi che riguardano l'inserimento degli articoli determinativi e quelli indeterminativi negli appositi spazi. Un punto rilevante di questa attività riguarda l'analisi dell'uso degli articoli relativamente alla categoria del genere e del numero e quella delle varianti morfo-fonologiche, quindi adattando gli articoli nel contesto in cui la lingua italiana lo richiede.

\subsection{Risultati}

Attività 1 - La produzione scritta

I dati relativi alla produzione scritta permettono di osservare imprecisioni al livello di accordo. Nei due test sono rilevati problemi legati all'accordo dell'articolo con il SN. Nella prima produzione scritta dove l'argomento era scelto dagli studenti 
abbiamo visto che la percentuale degli errori commessi era alta rispetto alla seconda produzione scritta in cui certe scelte sono state obbligate. Si può osservare dal graffico che è stato un miglioramento dalla prima produzione alla seconda. Questo a causa delle riflessioni fatte dopo la prima produzione, ma anche a causa delle parole chiave assegnate dal docente.

Graffico 1. I dati nelle due produzioni scritte

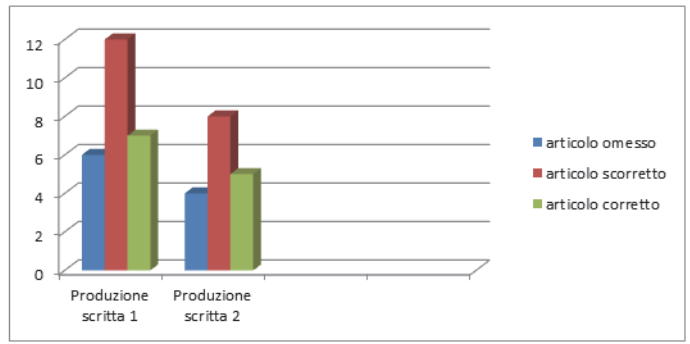

Come si può osservare nel graffico 1, le omissioni degli articoli diminuiscono nella seconda rilevazione dei dati, per quanto riguarda l'accordo all'interno del SN, osserviamo la presenza di numerose sostituzioni dell'articolo definito lo con il con il nome "studente", l'articolo non viene omesso ma sostituito dall'articolo il: il studente invece di lo studente; i con gli: $i$ amici invece di gli amici; i esercizi invece di gli esercizi; sovraestensioni dell'articolo un con uno o una: con il nome "studente" viene messo l'articolo un al posto di uno: un studente invece di uno studente, un professione invece di una professione; la tendenza di usare l'articolo definito sulla base di un'assonanza con la vocale finale del SN che segue: $i$ lezioni, le esame, la diploma invece di le lezioni, l'esame, il diploma; la tendenza di usare l'articolo con i possessivi seguiti dai nomi di parentela: *il mio padre, *la sua sorella, *il mio marito.

Attività 2 - Inserimento dell'articolo nel contesto.

Tabella 1. L'accordo dell'articolo con la SN

\begin{tabular}{|c|c|c|c|c|c|}
\hline tipo esempio & $\begin{array}{l}\text { genenere } \\
\text { numero }\end{array}$ & $\begin{array}{l}\text { desinenza } \\
\text { nominale }\end{array}$ & articolo & $\begin{array}{l}\text { corretto } \\
\text { (n) }\end{array}$ & $\begin{array}{c}\text { scorretto } \\
\text { (n) }\end{array}$ \\
\hline def. amico & $\mathrm{m} / \mathrm{sg}$ & -0 & $1^{\prime}$ (lo) & 15 & 5 \\
\hline def. automobile & $\mathrm{f} / \mathrm{sg}$ & $-e$ & $1^{\prime}(1 \mathrm{a})$ & 14 & 6 \\
\hline def. frasi & f/pl. & $-\mathrm{i}$ & le & 10 & 10 \\
\hline def. mano & $\mathrm{f} / \mathrm{sg}$. & -0 & la & 17 & 3 \\
\hline def. moglie & f/sg. & $-\mathrm{e}$ & la & 17 & 3 \\
\hline def. occhi & $\mathrm{m} / \mathrm{pl}$. & $-\mathrm{i}$ & gli & 10 & 10 \\
\hline def. panorama & $\mathrm{m} / \mathrm{sg}$. & $-\mathrm{a}$ & il & 6 & 14 \\
\hline def. situazione & $\mathrm{f} / \mathrm{sg}$ & $-\mathrm{e}$ & la & 14 & 6 \\
\hline -def. idea & $\mathrm{f} / \mathrm{sg}$. & $-\mathrm{a}$ & un ${ }^{\prime}$ & 12 & 8 \\
\hline - def. problema & $\mathrm{m} / \mathrm{sg}$. & $-a$ & un & 15 & 5 \\
\hline -def. zio & $\mathrm{m} / \mathrm{sg}$. & -0 & uno & 7 & 13 \\
\hline -def. televisione & $\mathrm{f} / \mathrm{sg}$. & $-\mathrm{e}$ & una & 14 & 6 \\
\hline -def. ora & f/sg. & $-a$ & $u^{\circ}$ & 13 & 7 \\
\hline -def. notte & f/sg. & $-e$ & una & 15 & 5 \\
\hline -def. albergo & $\mathrm{m} / \mathrm{sg}$. & -0 & un & 17 & 3 \\
\hline -def. spettacolo & $\mathrm{m} / \mathrm{sg}$. & -0 & uno & 7 & 13 \\
\hline
\end{tabular}

Come si evince dalla tabella, la maggior parte degli errori, sono quindi dovuti alla scelta dell'articolo. Si può dire che gli apprendenti abbiano scelto le desinenze più probabili considerando il genere e il numero dei nomi (*la panorama, *i frasi, *le situazione). La tendenza dominante è però l'uso degli allomorfi più comuni, vale a dire $i$, il, un ( ${ }^{*} i$ occhi, *un idea/ora), oppure il cambiamento del genere ( ${ }^{*} i$ automobile, *un televisione). Ci sono anche casi in cui uno e una sono sostituiti da un ('un spettacolo, *un zio, ${ }^{*}$ un notte) e un caso in cui un è sostituito da un' ( ${ }^{\star} u n '$ amico).

\section{Conclusioni}


Nel presente lavoro si è messo in evidenza le difficoltà che uno studente albanofono può incontrare lungo il suo percorso di apprendimento dell'italiano come lingua straniera. Abbiamo osservato, inoltre, che la selezione dell'articolo è un compito molto difficile per un apprendente la cui L1 esprime la definitezza del SN con elementi diversi rispetto all'italiano. È stato notato che, gli apprendenti albanesi tendono ad omettere l'articolo in determinati contesti e incertezze al livello di accordo all'interno del SN con il genere e il numero. Inoltre, vediamo la tendenza di sostituire l'articolo definito con quello indefinito nel contesto linguistico e viceversa. Dunque, nella produzione scritta si vede una maggiore accuratezza nella scelta dell'articolo relative all'accordo e alla definitezza [-definitezza] che nell'inserimento dell'articolo definito 0 indefinito nel contesto dato.

\section{Bibliografia}

Agalliu, F., Angoni, E., Demiraj, Sh., Dhrimo, A., Hysa, E., Lafe, E., Likaj, E., (1995) Gramatika e Gjuhës Shqipe, Vëllimi I, Morfologjia, Tiranë.

Dardano, M., Trifone, P.(1995) Grammatica italiana con nozioni di linguistica. Bologna.

Hawkins, R., Mayo, M. del P. G. (2009) Second language acquisition of articles, John Benjamins Publishing Company, Amsterdam I Philadelphia

Kambarov, Z. (2008) The concept of definiteness and its application to automated reference to resolution, Peter Lang Publishing, Inc. New York.

Lyons C. (1999), Definiteness, Cambridge University Press, Cambridge.

Newmark, L., Hubbard, P., Prifti, P. (1982) Standard Albanian. A reference grammar for students. Stendford University Press, California.

Renzi, L. (1991) Grande grammatica italiana di consultazione, II Mulino, Bologna.

Renzi, L. (2001) L'articolo, in Renzi L.,Salvi G., Cardinaletti Grande grammatica di consultazione, Vol I, II Mulino, Bologna.

Riza, S. (1982) Probleme të nyjave të shqipes. In Studime filologjike, Tiranë, Nr.1.

Serianni, L. (1989) Grammatica italiana Italiano comune e lingua letteraria, Bologna, UTET Libreria, Torino.

Topalli, K. (2009) Nyjat e shqipes, Tiranë. 